\title{
Evaluation of protection against Chlamydophila abortus challenge after DNA immunization with the major outer-membrane protein-encoding gene in pregnant and non-pregnant mice
}

\author{
Céline Héchard, † Olivier Grépinet and Annie Rodolakis
}

Correspondence

Olivier Grépinet

grepinet@tours.inra.fr

Received 5 June 2002

Accepted 21 August 2002
Unité de Pathologie Infectieuse et Immunologie, INRA - Centre de Tours, 37380 Nouzilly, France

The protective effect of DNA vaccination with the gene encoding the major outer-membrane protein (MOMP) of Chlamydophila abortus has been studied in non-pregnant and pregnant mouse models after chlamydial challenge. OF1 outbred mice were vaccinated intramuscularly three times every 3 weeks, mated and challenged with $C$. abortus 2 weeks after the last injection of DNA. In nonpregnant mice, the MOMP DNA vaccine elicited a specific humoral response with predominantly IgG2a antibodies, suggesting a Th1-type immune response. The induced antibodies showed no in vitro neutralizing effect on C. abortus infectivity. Moreover, immunization with the momp gene showed no reduction in the mean splenic bacterial counts of non-pregnant or pregnant mice or in the mean placental bacterial counts of pregnant mice after the C. abortus challenge. Nevertheless, the MOMP DNA immunization induced a non-specific and partial protection in fetuses against challenge.

\section{INTRODUCTION}

One of the most common causes of considerable loss in breeding is the abortion of goats and sheep induced by the intracellular obligate bacterium Chlamydophila abortus (Chlamydia psittaci serotype 1) (Papp \& Shewen, 1996). Furthermore, these bacteria present a zoonotic risk to pregnant women, since several cases of human chlamydial abortion have been reported (Buxton, 1986). A live attenuated vaccine is currently used in small ruminants (Rodolakis, 1983). This vaccine is effective and safe but does not allow the detection of infected animals in vaccinated flocks.

The predominant component and immunodominant antigen of the Chlamydiaceae surface membrane, the $40 \mathrm{kDa}$ major outer-membrane protein (MOMP), presents four surface-exposed variable domains (VDI-VDIV) flanked by five conserved regions and exhibits an oligomeric form, probably trimeric, that functions as a porin (De Sa, 1996). In a murine model of C. abortus infection, protective immunity can be conferred by antibodies (Buzoni-Gatel et al., 1990) and, more particularly, by the passive transfer of specific antibodies to the $110 \mathrm{kDa}$ oligomeric MOMP (De Sa et al., 1995), so MOMP has been considered as the most likely vaccine candidate against $C$. abortus infections. Native MOMP (Pal et al., 1997; Tan et al., 1990), recombinant

†Present address: Laboratoire d'Immuno-dermatologie, Centre Médical Universitaire, 1 rue Michel Servet, $\mathrm{CH}-1211$ Genève 4, Switzerland.

Abbreviation: MOMP, major outer-membrane protein.
MOMP (Tuffrey et al., 1992), synthetic MOMP peptides (Su et al., 1995) and live recombinant vectors (Murdin et al., 1995) expressing momp genes from members of the Chlamydiaceae have been evaluated in different animal models. Limited immunity and protection were generated in animals when the vaccinal approaches attempted to preserve the conformational structure of MOMP. Nevertheless, most trials were unsuccessful. A recent study showed that protective immunity against a Chlamydia trachomatis genital challenge was induced with a vaccine based on the MOMP associated with lipophilic immune response-stimulating complexes (Igietseme \& Murdin, 2000).

DNA vaccination represents an exciting means to induce specific immunity to a single antigen and, consequently, fits in the marker vaccine family (van Oirschot et al., 1996). Indeed, the direct injection of a DNA plasmid encoding an immunogenic protein into animals has resulted in both humoral and cell-mediated specific immune responses (Donnelly et al., 1997). Since DNA vaccination preferentially elicits a Th1-type immune response, it appears to be particularly appropriate for preventing intracellular bacterial infection (Strugnell et al., 1997). This novel strategy of vaccination has been assessed in different animal models and seems suitable for veterinary medicine (Babiuk et al., 2000).

DNA vaccination with the momp gene has already been tested in C. trachomatis (Pal et al., 1999; Zhang et al., 1997), Chlamydophila pneumoniae (Penttilä et al., 2000) and 
Chlamydophila psittaci (Vanrompay et al., 1999). In contrast to that observed in the C. psittaci model, no or modest protection was observed in the $C$. trachomatis and $C$. pneumoniae models. A single attempt at DNA vaccination against $C$. abortus in an abortion model is described in the literature (Héchard et al., 2002). In this model, only a nonspecific and partial protection, probably due to the $\mathrm{CpG}$ motifs of bacterial DNA, was observed in pregnant mice immunized with a plasmid encoding the heat-shock protein DnaK.

The purpose of the present study was to evaluate the protective effect of intramuscular injection of the C. abortus AB7 momp gene in pregnant and non-pregnant mouse models. Protection was evaluated by chlamydial clearance in spleens, placentas and fetuses and the humoral response was analysed by measuring isotype-specific anti-MOMP antibodies.

\section{METHODS}

C. abortus strains. The virulent $C$. abortus strain $\mathrm{AB} 7$ isolated from an ovine abortion (Faye et al., 1972) and the vaccinal strain 1B obtained by selection of a temperature-sensitive mutant after nitrosoguanidine mutagenesis of C. abortus AB7 (Rodolakis, 1983) were used. Bacteria were propagated in yolk sacs of embryonated specific-pathogen-free chicken eggs inoculated at day 7 , purified as described previously (Caldwell et al., 1981) and stored at $-80{ }^{\circ} \mathrm{C}$.

Cloning of the mompgene. To produce the vaccinal vector, the momp gene was amplified by PCR and cloned into the vector pcDNA3.1 (Invitrogen) carrying the human cytomegalovirus immediate early promoter and the bovine growth hormone polyadenylation signal. The PCR was performed using chlamydial genomic DNA (80 ng) as template with dNTPs (200 $\mu \mathrm{M}$ each), specific primers ( $1 \mu \mathrm{M}$ each) and 1 U Pfu DNA polymerase (Promega) in a Perkin Elmer 9600 thermocycler with the following program: $94{ }^{\circ} \mathrm{C}$ for $5 \mathrm{~min}, 1$ cycle; 30 cycles of $50{ }^{\circ} \mathrm{C}$ for $45 \mathrm{~s}, 72{ }^{\circ} \mathrm{C}$ for $2 \mathrm{~min}$ and $94{ }^{\circ} \mathrm{C}$ for $45 \mathrm{~s}$ and 1 cycle of $50{ }^{\circ} \mathrm{C}$ for $45 \mathrm{~s}$ and $72{ }^{\circ} \mathrm{C}$ for $10 \mathrm{~min}$. Specific primers were determined according to the DNA sequence of $C$. abortus AB7 containing an EcoRI site (underlined) and Kozak sequence (bold) in the forward primer (MOMP-FW; 5'CTCGAATTCACCATGAAAAAACTCTTGAAATCG-3') and an XhoI site (underlined) in the reverse primer (MOMP-RV; 5'-CT CCTCGAGTTAGAATCTGAATTGAGCATTC-3'). The resulting fragment was inserted into the vector pcDNA3.1 after linearization by EcoRI/XhoI double digestion to generate pcDNA3.1::MOMP. The plasmid pcDNA3.1:: MOMP and the pcDNA3.1 control plasmid were purified after an overnight Luria-Bertani culture of recombinant Escherichia coli DH5 $\alpha$ using the EndoFree Plasmid Mega kit (Qiagen). Plasmid DNA was dissolved at $1 \mu \mathrm{g} \mathrm{l}^{-1}$ in endotoxin-free PBS (Sigma). The sequence of the vaccinal vector pcDNA3.1:: MOMP was verified by DNA sequencing.

Immunization and challenge. All the studies were carried out with 6week-old female outbred OF1 Swiss mice (IFFA Credo). Prior to DNA immunization, each mouse was injected with cardiotoxin (Latoxan) into the tibialis anterior muscles of both hind legs to enhance the uptake of plasmid DNA (Davis et al., 1993). Five days later, mice were anaesthetized by an intraperitoneal injection of ketamine and xylazine (respectively 80 and $8 \mathrm{mg} \mathrm{kg}^{-1}$ body weight) and immunized with pcDNA3.1:: MOMP plasmid by intramuscular injections $(50 \mu \mathrm{g}$ in each tibialis anterior). Mice were boosted in the same way at days 21 and 42. The negative-control mice were immunized intramuscularly with endotoxin-free PBS (virulence control) or pcDNA3.1 plasmid. Positive- control mice were immunized with one subcutaneous injection of $4 \times 10^{4}$ p.f.u. of the live attenuated $1 \mathrm{~B}$ vaccine at day 1 . Groups of 10 and 20 mice were similarly immunized for evaluation of the protective effect of the DNA vaccine and respectively constituted the non-pregnant and pregnant groups. The five groups of pregnant mice were mated at day 44. Non-pregnant and pregnant mice were challenged at day 58 by an intraperitoneal injection of $4 \times 10^{4}$ p.f.u. C. abortus AB7. One group of pregnant mice neither immunized nor challenged was kept as a control for the pregnancy (gestation control).

Antibody response. Blood samples were collected from the retroorbital sinus of non-pregnant mice for the detection of anti-MOMPspecific antibodies before each DNA injection (days 0, 19, 41), just before the challenge infection (day 56) and at day 63. Blood samples were stored overnight at room temperature and then centrifuged $(600 \mathrm{~g}$, $15 \mathrm{~min}, 4{ }^{\circ} \mathrm{C}$ ) and sera were collected and stored at $-20^{\circ} \mathrm{C}$. The specificity of anti-MOMP antibodies was checked by ELISA as described previously (Héchard et al., 2002). The recombinant MOMP used as antigen was kindly provided by Dr R. Ashley (University of Edinburgh, UK) (Wyllie et al., 1999). Recombinant MOMP (100 $\mu \mathrm{l})$, denatured by heating $\left(100{ }^{\circ} \mathrm{C}\right.$ for $\left.10 \mathrm{~min}\right)$ and diluted in $\mathrm{Mg}^{2+}$-free, $\mathrm{Ca}^{2+}$-free PBS $(\mathrm{pH} 7 \cdot 4)$ at a concentration of $1 \mu \mathrm{g} \mathrm{ml}^{-1}$, was used for coating microtitre plates (Nunc-Immuno Plate Maxisorp Surface) for $16 \mathrm{~h}$ at $4{ }^{\circ} \mathrm{C}$. Sera used for ELISA were serially diluted in $\mathrm{Mg}^{2+}$-free, $\mathrm{Ca}^{2+}$-free PBS (pH 7.4), $0 \cdot 05 \%$ Tween $20 ; 1: 200$ for the less reactive serum or starting from $1: 200$ to $1: 800$ for the more reactive serum.

Determination of antibody isotypes. Determination of antibody isotypes was carried out using the ELISA as described above. Briefly, after incubation with dilutions $(1: 50)$ of the pooled sera of pcDNA3.1:: MOMP-vaccinated mice collected at day 56 or 63 , the isotypes were revealed by adding $100 \mu$ l of a $1: 1000$ dilution of peroxidase-conjugated anti-mouse IgG1 or IgG2a (Nordic Immunological Laboratories). The $A_{414}$ was measured on a microplate reader (Multiskan RC, Thermo Labsystems) after $1 \mathrm{~h}$ of incubation with the substrate (ABTS) at room temperature.

In vitro neutralization assay in the presence of the complement. The neutralization assay was done by the plaque-reduction assay using McCoy cells (Schachter et al., 1974). Pooled sera of PBS-, pcDNA3.1and pcDNA3.1:: MOMP-vaccinated mice were diluted in $\mathrm{Mg}^{2+}$-free, $\mathrm{Ca}^{2+}$-free PBS (pH 7.4) containing $10 \%$ fetal bovine serum and $2 \cdot 5 \%$ guinea pig serum. Two hundred microlitres of these dilutions $\left(10^{-1}\right.$, $10^{-2}$ and $\left.10^{-3}\right)$ were added to an equal volume $(200 \mu \mathrm{l})$ of an AB7 chlamydial suspension $\left(10^{4}\right.$ p.f.u.), incubated for $2 \mathrm{~h}$ at $37^{\circ} \mathrm{C}$ and then titrated on McCoy cells by the plaque assay method (Rodolakis \& Chancerelle, 1977). The results are expressed for each serum as the percentage p.f.u. of the PBS control.

Evaluation of protection. The protective effect of the DNA vaccine was assessed by the evaluation of the number of living offspring per litter at birth or 8 days after birth and the clearance of bacteria in spleens, placentas and fetuses. After challenge, the outcome of pregnant mice was monitored daily. Mice were considered as protected when the number of living offspring per litter 8 days after birth was significantly different $(P<0.05)$ from the number for the virulence control as described previously (Rodolakis, 1983). All the non-pregnant mice and five mice of each group of pregnant mice were euthanized at day 63 . The spleens from non-pregnant mice and the uteruses and spleens from pregnant mice were removed aseptically. All the placentas from the same uterine horn were dissected from the fetuses. Placentas from the same uterine horn were pooled as well as the fetuses. Pooled placentas, pooled fetuses and spleens were weighed and frozen at $-80{ }^{\circ} \mathrm{C}$. Organs were homogenized in PBS with a glass grinder, diluted in PBS-DEAE dextran $(0.01 \%)$ and titrated by plaque assay on McCoy cells (Rodolakis \& Chancerelle, 1977). The course of infection was evaluated by counting 
the number of p.f.u. and expressed as $\log _{10}$ p.f.u. per organ for spleens and $\log _{10}$ p.f.u. per uterine horn for placentas and fetuses.

Statistical tests. Analysis of the results was performed using the software InStat 2.03 for Macintosh. For all protective results, the mean was calculated using a one-way analysis of variance and a comparison of the means was then carried out through a Student-Newman-Keuls multiple comparison test. The minimal statistical significance was judged at $P<0 \cdot 05$.

\section{RESULTS}

\section{Analysis of the humoral response}

The anti-MOMP IgG antibody response was assessed by ELISA (Fig. 1). The anti-MOMP IgG titre reached a maximum in the non-pregnant mice immunized with pcDNA3.1::MOMP after the third DNA injection (day 56) and decreased after challenge (day 63). This decrease in anti-MOMP IgG antibody titre at day 63 was also observed without challenge (data not shown). The substantial variations of the $\operatorname{IgG}$ titre in individual mice on the last two sampling days were probably due to the fact that the murine model used in these experiments was an outbred one. No MOMP-specific IgG antibody was identified in sera from non-immunized mice (data not shown) or from mice immunized with pcDNA3.1 (Fig. 1). The specificity of antiMOMP antibodies was also examined by immunoblotting. Sera collected from immunized mice before any DNA injection (day 0 ) or before challenge (day 56) and after challenge (day 63) were tested on crude extracts of $C$. abortus AB7 as well as on boiled recombinant MOMP. Since no MOMPspecific antibodies were detected (data not shown), we could conclude that the anti-MOMP antibody titre was probably too low to detect native or recombinant MOMP by immunoblotting.

The isotypes of anti-MOMP antibodies of sera collected at

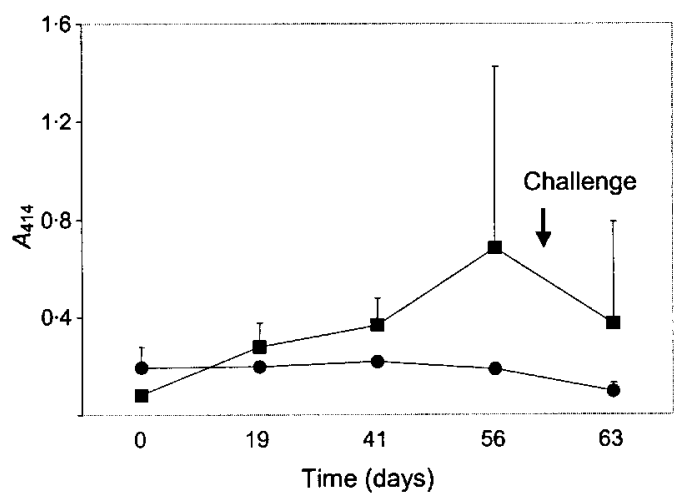

Fig. 1. Kinetics of the antibody response of DNA-immunized mice. Sera were collected at different times after immunization with

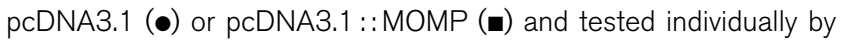
ELISA for titration of anti-MOMP-specific lgG antibodies. The mean $A_{414}$ (+SEM) is shown for each time-point. days 56 and 63 from pcDNA3.1:: MOMP-vaccinated mice were tested by ELISA. The predominant isotype was IgG2a (data not shown). No IgG1 was found.

The ability of the sera to neutralize the infectivity of the virulent $\mathrm{AB} 7$ strain of $C$. abortus was assessed by the plaquereduction test. The sera of $1 \mathrm{~B}$-vaccinated mice had a substantial neutralizing effect ( $90 \%$ at the $10^{-1}$ dilution; Fig. 2). while the sera of pcDNA3.1- and pcDNA3.1::MOMPvaccinated mice respectively had a modest neutralizing effect $(60 \%)$ and a non-significant neutralizing effect $(20 \%)$.

\section{Efficacy of DNA immunization on pregnancy}

As expected, the number of live newborn mice observed in the $1 \mathrm{~B}$ group was not significantly different from the number of live newborns observed in the gestation control group $(P>0 \cdot 05)$, but it was significantly higher $(P<0 \cdot 01)$ than the number monitored in the virulence group (Fig. 3). The numbers of living offspring at birth and 8 days after birth for the gestation control and $1 \mathrm{~B}$ groups were not significantly different. For the virulence group and the pcDNA3.1- and pcDNA3.1:: MOMP-vaccinated mice, the number of living offspring at birth was more than at 8 days after birth, but this difference was not significant. The numbers of live newborn in the virulence, pcDNA3.1 and pcDNA3.1:: MOMP groups were not significantly different $(P>0 \cdot 05)$, but were significantly lower $(P<0.05)$ than in the gestation control and 1B groups. Therefore, $\mathrm{pcDNA3}$.1:: MOMP-vaccinated mice were not protected against abortion due to C. abortus $\mathrm{AB} 7$ challenge.

Protection was also evaluated at a placental and fetal level by titration of $C$. abortus in these organs. Chlamydial titres in placentas were higher than in fetuses $(P<0.001)$. As expected, chlamydial titres in the placentas and fetuses of $1 \mathrm{~B}$-vaccinated mice were significantly lower $(P<0 \cdot 01)$ than in the virulence group (Fig. 4). 1B-vaccinated mice were consequently protected against chlamydial infection. Chlamydial titres in the placentas of pcDNA3.1- and pcDNA3.1:: MOMP-vaccinated mice were not significantly

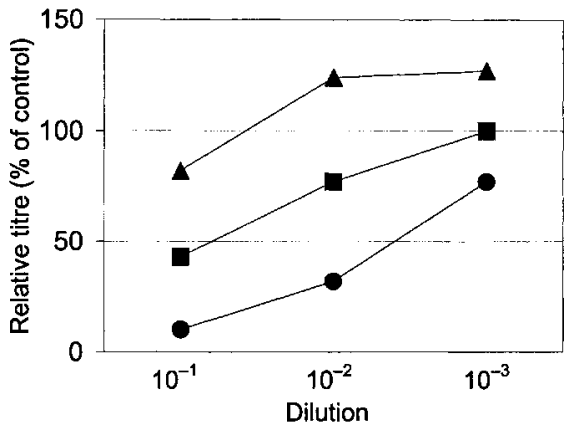

Fig. 2. Neutralizing effect of sera from 1B- (•), pcDNA3.1- (घ) or pcDNA3.1 : :MOMP- $(\mathbf{A})$ vaccinated mice. Mouse sera collected at day 56 were diluted $10^{-1}, 10^{-2}$ or $10^{-3}$ and then added to the chlamydial suspension. These solutions had been titrated and the results are expressed relative to the control. 


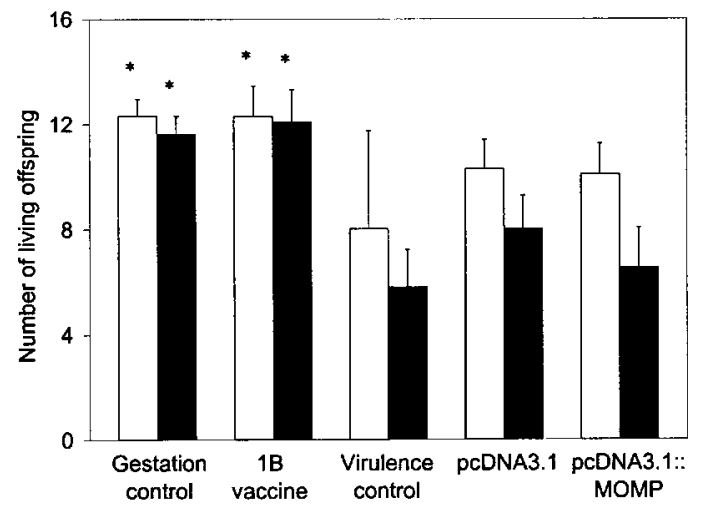

Fig. 3. Effect of DNA vaccination on viable offspring at birth (open bars) or 8 days after birth (filled bars). The gestation control group was neither infected nor immunized and the virulence group was infected but not immunized. Mice were immunized with pcDNA3.1 or pcDNA3.1 : : MOMP or with the $1 \mathrm{~B}$ vaccine. After immunization, mice were infected at 12 days of gestation. Results are expressed as mean numbers of living offspring 1 week after birth (+SEM). *, Significantly different from the respective virulence control $(P<0.05)$.



Fig. 4. Chlamydial clearance in placentas (open bars) and fetuses (filled bars) of pregnant mice. Mice were immunized and mated and then challenged with $C$. abortus AB7 and euthanized 5 days after challenge. Quantities of bacteria in placentas and fetuses are expressed as $\log _{10}$ p.f.u. per uterine horn (+SEM). *, Significantly different from the respective virulence control $(P<0.05)$.

different from those of the virulence group $(P>0 \cdot 05)$. This would indicate that DNA vaccination did not elicit sufficient protection against placental colonization. Nevertheless, the fetuses of pcDNA3.1- and pcDNA3.1:: MOMP-vaccinated mice were significantly less infected than those of the virulence group $(P<0 \cdot 05)$ (Fig. 4). Thus, partial protection was observed in fetuses of 1B-, pcDNA3.1- and pc DNA3.1:: MOMP-vaccinated mice.

\section{Clearance of $C$. abortus AB7 from the spleen}

The induced protection was evaluated by splenic titration of C. abortus on pregnant and non-pregnant mice. As expected, chlamydial titres in spleens from non-pregnant or pregnant $1 \mathrm{~B}$-vaccinated mice were significantly lower $(P<0.001$ and $P<0 \cdot 01$, respectively) than in the virulence group. Thus, $1 \mathrm{~B}$-vaccinated mice were protected against chlamydial infection (Fig. 5). Chlamydial titres in the spleens from pcDNA3.1- and pcDNA3.1:: MOMP-vaccinated, non-pregnant or pregnant mice were not significantly different from those from mice of the virulence group $(P>0 \cdot 05)$. These results suggested that DNA vaccination did not induce splenic protection in pregnant or non-pregnant mice.

\section{DISCUSSION}

In veterinary medicine, vaccines need to overcome a large number of hurdles, including the cost of production, the ease of delivery, safety and the complete protection of animals against disease. DNA vaccination has recently been tested against many pathogens and is promising in veterinary medicine (Babiuk et al., 2000). The aim of this study was to investigate the protective effect of DNA vaccination with the MOMP-encoding gene against C. abortus challenge in a murine model. The mouse model usually employed for the evaluation of C. abortus virulence (Rodolakis et al., 1989) as well as vaccine efficiency (Rodolakis, 1983) was the OF1 outbred mouse model of abortion. This model presented various advantages. It was easy to perform, since it consisted of evaluation of the living offspring per mouse. Moreover, the protective effects of vaccines obtained in OF1 outbred mice were correlated with those observed in the ovine model. Finally, this mouse model has been used successfully in the past to evaluate the efficiency of $1 \mathrm{~B}$ vaccine and is now taken as a reference (Rodolakis, 1983). Nevertheless, this OF1 mouse model presented two major disadvantages. Firstly, as it involves outbred mice, this model is not suitable for

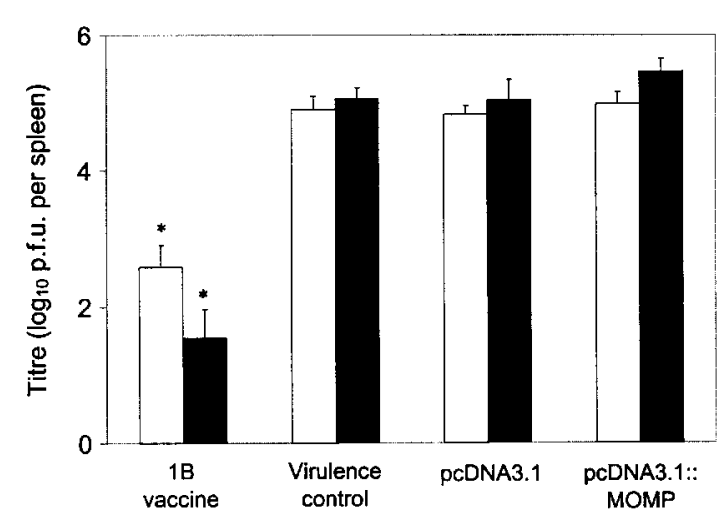

Fig. 5. Chlamydial clearance in spleens of DNA-immunized pregnant (open bars) or non-pregnant (filled bars) mice. Mice were immunized by three intramuscular injections of pcDNA3.1 or pcDNA3.1 : : MOMP or one injection of the $1 \mathrm{~B}$ vaccine. Mice were then challenged with $C$. abortus AB7 and euthanized 5 days after challenge. Quantities of bacteria in spleens are expressed as $\log _{10}$ p.f.u. per spleen (+SEM). *, Significantly different from the respective virulence control $(P<0.05)$. 
evaluation of the cellular immune response. Secondly, it can be weakly discriminatory, since a small difference in the titre of the C. abortus challenge (nearness abortive dose $50 \%$ ) can modify the number of living offspring very significantly. Together with the high variability between the results obtained with outbred mice, this could have made the protection observed non-significant. Thus, the comparable mean numbers of living offspring obtained from pcDNA3.1and pcDNA3.1::MOMP-vaccinated mice drove us to investigate more precisely the protection in other models of infection evaluation, including the splenic, placental and fetus bacterial burden inquiries.

Although DNA immunization with the momp gene elicited production of MOMP in vivo, since it induced specific antiMOMP antibodies, it failed to reduce the abortive effect of $C$. abortus challenge. No protection was observed after MOMP DNA immunization in either the placental colonization or splenic colonization model. However, partial reduction of infection was observed in fetuses of pcDNA3.1- and pcDNA3.1::MOMP-vaccinated mice. As placentas were infected, fetuses were probably protected by a barrier effect, but this protective effect was not sufficient to allow the survival of living offspring. This non-specific and partial protection could be explained by the immunostimulating properties of the $\mathrm{CpG}$ sequences present in the vaccinal plasmid backbone (Krieg, 2000). These unmethylated CpG motifs are recognized as a danger signal by the vertebrate immune system and trigger a Th1-biased immune response with IgG2a antibody production. This hypothesis is therefore in agreement with the predominant IgG2a isotype of antiMOMP antibodies found in our experiments. Analysis of cytokine profiles could have confirmed this Th1-like immune response. Buendía et al. (1999) reported high levels of interferon- $\gamma$ and interleukin- 12 in the serum of mice infected with the $C$. abortus strains $\mathrm{AB} 7$ and $1 \mathrm{~B}$, suggesting strongly that effective resolution of the infection involves a Th1-like immune response.

The increased mortality of newborn mice observed in the pcDNA3.1 and pcDNA3.1::MOMP groups 8 days after birth could result from the loss of the nutritious properties of infected placentas, leading to frail living offspring, resulting in death even in the absence of fetal infection. Also, late contamination could be possible when the mice gave birth. Indeed, it has been reported that C. abortus was excreted when ewes gave birth and that they could therefore infect newborns as well as other ewes at this time (Rodolakis \& Souriau, 1979). This hypothesis could be transposable in the mouse model, since the number of living offspring appeared to be higher at birth than 8 days after birth. We could also propose that the newborn of $1 \mathrm{~B}$-vaccinated mice would be protected by the transfer of maternal antibodies via the milk, since the sera of these mice showed neutralizing properties.

In contrast with the pooled sera of $1 \mathrm{~B}$-vaccinated mice, the sera of pcDNA3.1- and pcDNA3.1::MOMP-vaccinated mice failed to neutralize chlamydial penetration in cells. As protective anti-MOMP antibodies are known to be directed against conformational epitopes (De Sa et al., 1995), we could speculate that the MOMP DNA vaccine induced linear epitope-specific antibodies. Probably due to the weak humoral response induced by the DNA vaccination, we failed to detect either conformational or linear MOMP after immunoblotting on whole $C$. abortus extracts. Another observation came from the weak level of anti-MOMP antibodies induced by the MOMP DNA vaccine. Because induction of the immune response is known to be linked directly to protein expression following DNA immunization (Barry \& Johnston, 1997), we supposed that MOMP expression in vivo was likely to be insufficient to induce a relevant level of antiMOMP antibodies. This was consistent with the fact that the antibodies of pcDNA3.1::MOMP-vaccinated mice sera failed to detect MOMP by immunoblotting, a less sensitive technique compared with ELISA.

Different methods have been evaluated in an attempt to increase the immune response generated by DNA vaccines (Gurunathan et al., 2000). More particularly, the vaccination protocol could be improved, as has already been done in a $C$. trachomatis model (Dong-Ji et al., 2000), using a DNA prime and a protein boost strategy known to enhance both humoral immune response and protection (Song et al., 2000). In addition, it might be possible to amplify the protective immune response by co-administration of an immunoregulatory cytokine-encoding plasmid with the vaccinal plasmid (Gurunathan et al., 2000). A protein boost and the coadministration of cytokine-encoding plasmids can also be combined in order to achieve protection (Scheerlinck et al., 2001).

In summary, DNA vaccination with a plasmid vector encoding the momp gene of $C$. abortus elicited a weak humoral response, with the $\operatorname{IgG} 2 \mathrm{a}$ isotype predominant. Despite the antigenic properties of MOMP, DNA vaccination failed to protect pregnant mice from abortion against $C$. abortus challenge. In contrast, fetuses from DNA-vaccinated mice were non-specifically and partially protected. Therefore, it would be of interest to optimize the vaccination protocol and to use genetic adjuvants including cytokines or co-stimulating molecule genes in order to enhance the immune response.

\section{ACKNOWLEDGEMENTS}

The authors thank Dr Dominique Buzoni-Gatel for helpful discussion and critical reading of this manuscript and Françoise Bernard for excellent technical assistance. This work was supported by a grant from INRA-Région Centre.

\section{REFERENCES}

Babiuk, L. A., Babiuk, S. L., Loehr, B. I. \& van Drunnen Littel-van den Hurk, S. (2000). Nucleic acid vaccines: research tool or commercial reality. Vet Immunol Immunopathol 76, 1-23.

Barry, M. A. \& Johnston, S. A. (1997). Biological features of genetic immunization. Vaccine 15, 788-791.

Buendia, A. J., Sànchez, J., Del Rio, L., Garces, B., Gallego, M. C., Caro, 
M. R., Bernabe, A. \& Salinas, J. (1999). Differences in the immune response against ruminant chlamydial strains in a murine model. Vet Res 30, 495-507.

Buxton, D. (1986). Potential danger to pregnant women of Chlamydia psittaci from sheep. Vet Rec 118, 510-511.

Buzoni-Gatel, D., Bernard, F., Andersen, A. \& Rodolakis, A. (1990). Protective effect of polyclonal and monoclonal antibodies against abortion in mice infected by Chlamydia psittaci. Vaccine 8, 342-346.

Caldwell, H. D., Kromhout, J. \& Schachter, J. (1981). Purification and partial characterization of the major outer membrane protein of Chlamydia trachomatis. Infect Immun 31, 1161-1176.

Davis, H. L., Whalen, R. G. \& Demeneix, B. A. (1993). Direct gene transfer into skeletal muscle in vivo: factors affecting efficiency of transfer and stability of expression. Hum Gene Ther 4, 151-159.

De Sa, C. (1996). The major outer-membrane protein of Chlamydia: structure and functions. Vet Res 27, 317-331 (in French).

De Sa, C., Souriau, A., Bernard, F., Salinas, J. \& Rodolakis, A. (1995). An oligomer of the major outer membrane protein of Chlamydia psittaci is recognized by monoclonal antibodies which protect mice from abortion. Infect Immun 63, 4912-4916.

Dong-Ji, Z., Yang, X., Shen, C., Lu, H., Murdin, A. \& Brunham, R. C. (2000). Priming with Chlamydia trachomatis major outer membrane protein (MOMP) DNA followed by MOMP ISCOM boosting enhances protection and is associated with increased immunoglobulin $A$ and Th1 cellular immune responses. Infect Immun 68, 3074-3078.

Donnelly, J. J., Ulmer, J. B., Shiver, J. W. \& Liu, M. A. (1997). DNA vaccines. Annu Rev Immunol 15, 617-648.

Faye, P., Charton, A., Mage, C., Bernard, C. \& Le Layec, C. (1972). Propriétés hémagglutinantes du 'virus' de l'avortement enzootique des petits ruminants (souches de 'Rakeia' d'origine ovine et caprine). Note préliminaire. Bull Acad Vét Fr 45, 169-173.

Gurunathan, S., Klinman, D. M. \& Seder, R. A. (2000). DNA vaccines: immunology, application, and optimization. Annu Rev Immunol 18, 927-974.

Héchard, C., Grépinet, O. \& Rodolakis, A. (2002). Protection evaluation against Chlamydophila abortus challenge by DNA vaccination with a $d n a K$-encoding plasmid in pregnant and non-pregnant mice. Vet Res 33, 313-326.

Igietseme, J. U. \& Murdin, A. (2000). Induction of protective immunity against Chlamydia trachomatis genital infection by a vaccine based on major outer membrane protein-lipophilic immune response-stimulating complexes. Infect Immun 68, 6798-6806.

Krieg, A. M. (2000). Immune effects and mechanisms of action of CpG motifs. Vaccine 19, 618-622.

Murdin, A. D., Su, H., Klein, M. H. \& Caldwell, H. D. (1995). Poliovirus hybrids expressing neutralization epitopes from variable domains I and IV of the major outer membrane protein of Chlamydia trachomatis elicit broadly cross-reactive $C$. trachomatis-neutralizing antibodies. Infect Immun 63, 1116-1121.

Pal, S., Theodor, I., Peterson, E. M. \& de la Maza, L. M. (1997). Immunization with an acellular vaccine consisting of the outer membrane complex of Chlamydia trachomatis induces protection against a genital challenge. Infect Immun 65, 3361-3369.

Pal, S., Barnhart, K. M., Wei, Q., Abai, A. M., Peterson, E. M. \& de la Maza, L. M. (1999). Vaccination of mice with DNA plasmids coding for the Chlamydia trachomatis major outer membrane protein elicits an immune response but fails to protect against a genital challenge. Vaccine 17, 459-465.

Papp, J. R. \& Shewen, P. E. (1996). Localization of chronic Chlamydia psittaci infection in the reproductive tract of sheep. J Infect Dis 174, $1296-1302$.
Penttilä, T., Vuola, J. M., Puurula, V., Anttila, M., Sarvas, M., Rautonen, N., Makela, P. H. \& Puolakkainen, M. (2000). Immunity to Chlamydia pneumoniae induced by vaccination with DNA vectors expressing a cytoplasmic protein (Hsp60) or outer membrane proteins (MOMP and Omp2). Vaccine 19, 1256-1265.

Rodolakis, A. (1983). In vitro and in vivo properties of chemically induced temperature-sensitive mutants of Chlamydia psittaci var. ovis: screening in a murine model. Infect Immun 42, 525-530.

Rodolakis, A. \& Chancerelle, L. (1977). Plaque assay for Chlamydia psittaci in tissue samples. Ann Microbiol (Paris) 128B, 81-85 (in French).

Rodolakis, A. \& Souriau, A. (1979). Clinical evaluation of a commercial vaccine against chlamydial abortion of ewes. Ann Rech Vét 10, 41-48.

Rodolakis, A., Bernard, F. \& Lantier, F. (1989). Mouse models for evaluation of virulence of Chlamydia psittaci isolated from ruminants. Res Vet Sci 46, 34-39.

Schachter, J., Banks, J., Sugg, N., Sung, M., Storz, J. \& Meyer, K. F. (1974). Serotyping of Chlamydia. I. Isolates of ovine origin. Infect Immun 9, 92-94.

Scheerlinck, J. P., Casey, G., McWaters, P., Kelly, J., Woollard, D., Lightowlers, M. W., Tennent, J. M. \& Chaplin, P. J. (2001). The immune response to a DNA vaccine can be modulated by co-delivery of cytokine genes using a DNA prime-protein boost strategy. Vaccine 19, 4053-4060.

Song, M. K., Lee, S. W., Suh, Y. S., Lee, K. J. \& Sung, Y. C. (2000). Enhancement of immunoglobulin G2a and cytotoxic T-lymphocyte responses by a booster immunization with recombinant hepatitis $\mathrm{C}$ virus E2 protein in E2 DNA-primed mice. J Virol 74, 2920-2925.

Strugnell, R. A., Drew, D., Mercieca, J., DiNatale, S., Firez, N., Dunston, S. J., Simmons, C. P. \& Vadolas, J. (1997). DNA vaccines for bacterial infections. Immunol Cell Biol 75, 364-369.

Su, H., Parnell, M. \& Caldwell, H. D. (1995). Protective efficacy of a parenterally administered MOMP-derived synthetic oligopeptide vaccine in a murine model of Chlamydia trachomatis genital tract infection: serum neutralizing IgG antibodies do not protect against chlamydial genital tract infection. Vaccine 13, 1023-1032.

Tan, T. W., Herring, A. J., Anderson, I. E. \& Jones, G. E. (1990). Protection of sheep against Chlamydia psittaci infection with a subcellular vaccine containing the major outer membrane protein. Infect Immun 58, 3101-3108.

Tuffrey, M., Alexander, F., Conlan, W., Woods, C. \& Ward, M. (1992). Heterotypic protection of mice against chlamydial salpingitis and colonization of the lower genital tract with a human serovar $F$ isolate of Chlamydia trachomatis by prior immunization with recombinant serovar L1 major outer-membrane protein. J Gen Microbiol 138, $1707-1715$.

van Oirschot, J. T., Kaashoek, M. J. \& Rijsewijk, F. A. (1996). Advances in the development and evaluation of bovine herpesvirus 1 vaccines. Vet Microbiol 53, 43-54.

Vanrompay, D., Cox, E., Vandenbussche, F., Volckaert, G. \& Goddeeris, B. (1999). Protection of turkeys against Chlamydia psittaci challenge by gene gun-based DNA immunizations. Vaccine 17, $2628-2635$.

Wyllie, S., Longbottom, D., Herring, A. J. \& Ashley, R. H. (1999). Single channel analysis of recombinant major outer membrane protein porins from Chlamydia psittaci and Chlamydia pneumoniae. FEBS Lett $\mathbf{4 4 5}$, 192-196.

Zhang, D.-J., Yang, X., Berry, J., Shen, C., McClarty, G. \& Brunham, R. C. (1997). DNA vaccination with the major outer-membrane protein gene induces acquired immunity to Chlamydia trachomatis (mouse pneumonitis) infection. J Infect Dis 176, 1035-1040. 